\title{
Suprascapular block for chronic shoulder pain, retrospective evaluation of results
}

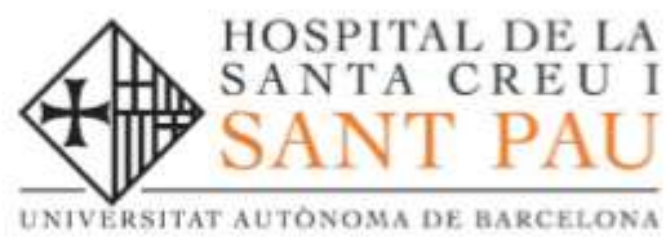

\section{A. Bainac Albadalejo1, R. Diaz Jover1, M. Revuelta Rizo1, A. Parera Ruiz ${ }^{1}$, M. Melo Cruz$^{1}$, E. Català Puigbò 1 \\ ${ }^{1}$ Hospital de la Santa Creu i Sant Pau - Barcelona (Spain)}

\section{Background}

To provide pain relief and to facilitate functional rehabilitation of patients suffering chronic shoulder pain (CSP), suprascapular nerve block (SSNB) can be made as a useful treatment. The objective of our study is to retrospectively evaluate the analgesic effect of SSNB with local anesthetics combined with corticoids and pulsed radiofrequency (PRF).

\section{Material_and methods}

We studied retrospectively a cohort of 50 consecutive patients receiving suprascapular nerve block for chronic shoulder pain between January 2014 and January 2017. We evaluate demographic characteristics and Numerical Rating Scale (NRS) before and after nerve block at 1 month and 3 months. We consider significant pain relief if reduction of NRS is $>30 \%$.

\section{Results and discussion}

Demographic characteristics; mean age $76 \pm 3$ years old, $82 \%$ of women and $18 \%$ men

Pain relief evaluated at 1 and 3 month are showed in figure $1 \& 2$

10

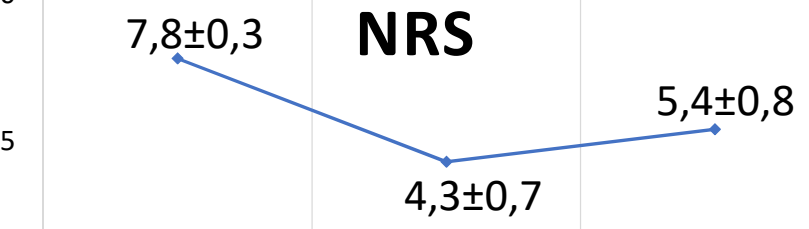

0 BEFORE TECHNIQUE 1 MONTH 3 MONTH Figure 1

\section{TECHNIQUE}

- Patient was placed in sitting position.

- Under sterile conditions, and after skin and soft tissues were anesthetized with $1 \mathrm{ml} 1 \%$ lidocaine.

- Under ultrasonographic view 23G radiofrequency needle with $5 \mathrm{~mm}$ active tip (Electrode CR-6, Cosman Medical, Burlington, USA) was advanced until suprascapular notch position was obtained.

- Sensory and motor stimulation at $50 \mathrm{~Hz}$ and $2 \mathrm{~Hz}$ respectively was performed. Stimulation threshold was required to be less than $0.6 \mathrm{~V}$ to identify nerve position.

- PRF was performed for 240 seconds and after a mixture of ropivacaine $0,2 \%(4 \mathrm{ml})+$ triamcinolone $40 \mathrm{mg}$ were injected

\section{SIGNIFICANT PAIN RELIEVE (NRS>30\%)}

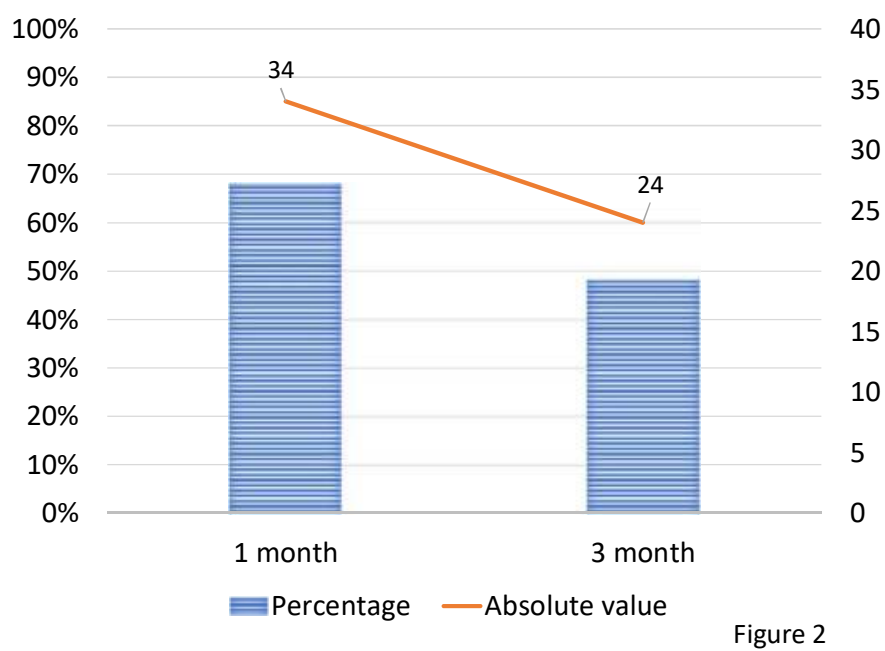

\section{Conclusions}

The use of SSNB with local anesthetics and corticoids combined with PRF achieves a reduction of NRS for 12 weeks in our patients with no complications reported. One of our goals is relief pain to facilitate physiotherapy and improve range of movement, this is possible due to SSNB provides fast analgesia in movement. However, we cannot conclude that it was the corticoid or PRF that caused the improvement. Further studies are needed to confirm these results.

\section{References}

Liu A, Zhang W, Sun M, Ma C, Yan S. Evidence-based Status of Pulsed Radiofrequency Treatment for Patients with Shoulder Pain: A Systematic Review of Randomized Controlled Trials. Pain Practice, 16, 4 (2016) 518-525 